\title{
The Protein Corona Mediates the Impact of Nanomaterials and Slows Amyloid Beta Fibrillation
}

\author{
Morteza Mahmoudi, ${ }^{\left[{ }^{[a, b]}\right.}$ Marco P. Monopoli, ${ }^{[c]}$ Meisam Rezaei, ${ }^{[a]}$ Iseult Lynch, ${ }^{[c]}$ \\ Filippo Bertoli, ${ }^{[c]}$ Jennifer J. McManus, ${ }^{*[d]}$ and Kenneth A. Dawson ${ }^{*[c]}$
}

The formation of protein fibrils is a consistent feature in the pathogenesis of several neurodegenerative diseases. The use of nanoparticles as theranostic agents to inhibit fibril formation has been suggested as a potential treatment for these socalled amyloid diseases. Protein fibrillation is defined as the process by which partially or fully unfolded proteins form beta-strand-stacked structures leading to formation of large insoluble linear aggregates or amyloid. ${ }^{[1]}$ Many proteins and peptides form amyloid-type structures, including amyloid beta (A $\beta$ ) peptide, ${ }^{[2]}$ prion protein, ${ }^{[3]} \alpha$-synuclein, ${ }^{[4]}$ polyglutamine, ${ }^{[5]}$ glucagon, ${ }^{[6]}$ and $\beta_{2}$-microglobulin. ${ }^{[7]}$

Amyloid beta deposits are found between neurons in patients with Alzheimer's disease, and have therefore received much attention. $A \beta$ is also widely used as a model protein to investigate the effect of nanomaterials (NMs) on the fibrillogenesis of proteins. ${ }^{[8]} A \beta$, which has a molecular weight of $\sim 4 \mathrm{kDa}$, is an amphipathic polypeptide prone to self-association and fibril formation. ${ }^{[9]}$ Monomeric $A \beta$, is an unstructured peptide that is soluble under physiological conditions, ${ }^{[10]}$ however, the fibrillar form has a characteristic cross- $\beta$ structure with stacking of $\beta$ strands perpendicular to the long axis of the fiber. ${ }^{[10]}$ While the fibrillation process can be affected by the presence of NMs, the acceleratory or inhibitory effect is highly dependent on the physicochemical properties of the NM and on the protein characteristics. ${ }^{[11]}$

It is now well recognized that the surfaces of NMs become covered with a condensed layer of biomolecules, called a protein corona, upon exposure to biological media. ${ }^{[12]}$ The exact composition of proteins in the corona is dependent on the specific surface properties of the NM, as only proteins with

[a] Prof. M. Mahmoudi, M. Rezaei

Department of Nanotechnology, Faculty of Pharmacy

Tehran University of Medical Sciences

Engelab Street, 14155-6451 Tehran (Iran)

E-mail:Mahmoudi@biospion.com

[b] Prof. M. Mahmoudi

Nanotechnology Research Center, Faculty of Pharmacy

Tehran University of Medical Sciences

Engelab Street, 14155-6451 Tehran (Iran)

[c] M. P. Monopoli, I. Lynch, F. Bertoli, Prof. K. A. Dawson

Centre for BioNano Interactions

Department of Chemistry and Chemical Biology, University College Dublin Belfield, Dublin 4 (Ireland)

E-mail: Kenneth.A.Dawson@cbni.ucd.ie

[d] Dr. J. J. McManus

Chemistry Department, National University of Ireland Maynooth

Maynooth, Co. Kildare (Ireland)

E-mail:jennifer.mcmanus@nuim.ie

Supporting information for this article is available on the WWW under http://dx.doi.org/10.1002/cbic.201300007. a strong affinity towards the NM surface will remain in the corona, eventually displacing proteins with lower affinity. The NM protein corona has been shown to last sufficiently long that the pristine surface of the NM is unlikely to ever be exposed in the biological milieu. NMs of various surface chemistries and sizes have been shown to both accelerate and inhibit $A \beta$ fibrillation in solution. ${ }^{[8,11 a, 13]}$ Direct interaction of the $A \beta$ peptide with the surface of the NM occurred in both cases. Where the NM promoted fibril formation, nucleation was accelerated on the surface, increasing the rate at which fibrils formed. When the NM inhibited formation, small oligomeric forms of $A \beta$ were present on the surface; this reduced the solution concentration and thus the probability for formation of a critical nucleus, and therefore the rate of fibril formation was slowed. Although these are important observations, in order to understand the fate of $A \beta$ exposed to NMs in a biological context, we must assess the interactions with NMs in the state in which they encounter $A \beta$ : we know that this will not be as a pristine NM, but as a NM bearing a protein corona. Thus an important step forward in developing our understanding of $\mathrm{A} \beta-\mathrm{NM}$ interactions in more biologically relevant conditions is to consider the case where the NM surface is already precovered with proteins and thus potentially less accessible to the $A \beta$, that is, to study the effect of protein-corona-coated NMs on $A \beta$ fibrillation.

Here we investigate the effects of protein-coated NMs on the fibrillation of $A \beta$, and discuss how the protein corona shell has an inhibitory effect on $A \beta$ fibrillation, regardless of the NM physicochemical properties (e.g., composition, size, shape (aspect ratio), and surface properties). Several different NMs (silica (100 and $200 \mathrm{~nm}$ ), polystyrene with carboxyl surface modification $(100 \mathrm{~nm})$, and multi-walled carbon nanotubes (CNT; diameter: 10-40 nm and length: 0.1-10 $\mu \mathrm{m}$; see Figure S1 and Table S1 in the Supporting Information)) were employed in this study and their effects on the $A \beta$ fibrillation process were probed in the absence and presence of a protein corona, as well as well as under conditions under which the protein corona was denatured.

The protein-coated NMs were prepared by incubating the NMs with plasma proteins at protein concentrations of $10 \%$ (in vitro simulated medium) and $100 \%$ (in vivo simulated medium, see the Supporting Information for details) for $1 \mathrm{~h}$ to allow proteins to condense onto the NM surface. This was followed by several washing steps to remove the loosely attached proteins from the surface of the NMs and leave only the strongly bound "hard corona" proteins attached to the NMs. In experiments to measure fibrillation, the NMs (bare and protein-coated; NM concentrations were fixed at $100 \mu \mathrm{g} \mathrm{mL}^{-1}$ ) 
were mixed with $A \beta(5,20$ or $40 \mu \mathrm{M}$ depending on the experiment), and $A \beta$ fibril formation was measured using the Thioflavin T (ThT) assay. ThT binds primarily to protein fibrils and not to oligomeric forms which precede fibril formation. A typical ThT assay is therefore characterized by a lag time, where nucleation and oligomerization of $A \beta$ occur, followed by the appearance of protein fibrils (observed as an increase in ThT fluorescence). Figure 1 shows the results of ThT assays for $A \beta$ in the presence of NMs of varying composition, size aspect ratio and surface charge, with and without protein coronas (formed at 10 or $100 \%$ plasma) and Figure S2 shows the reproducibility of the data.

These measurements show that all "bare" NMs used for the study accelerate the rate of $A \beta$ fibril formation, in agreement with literature. However when the NMs were precoated with
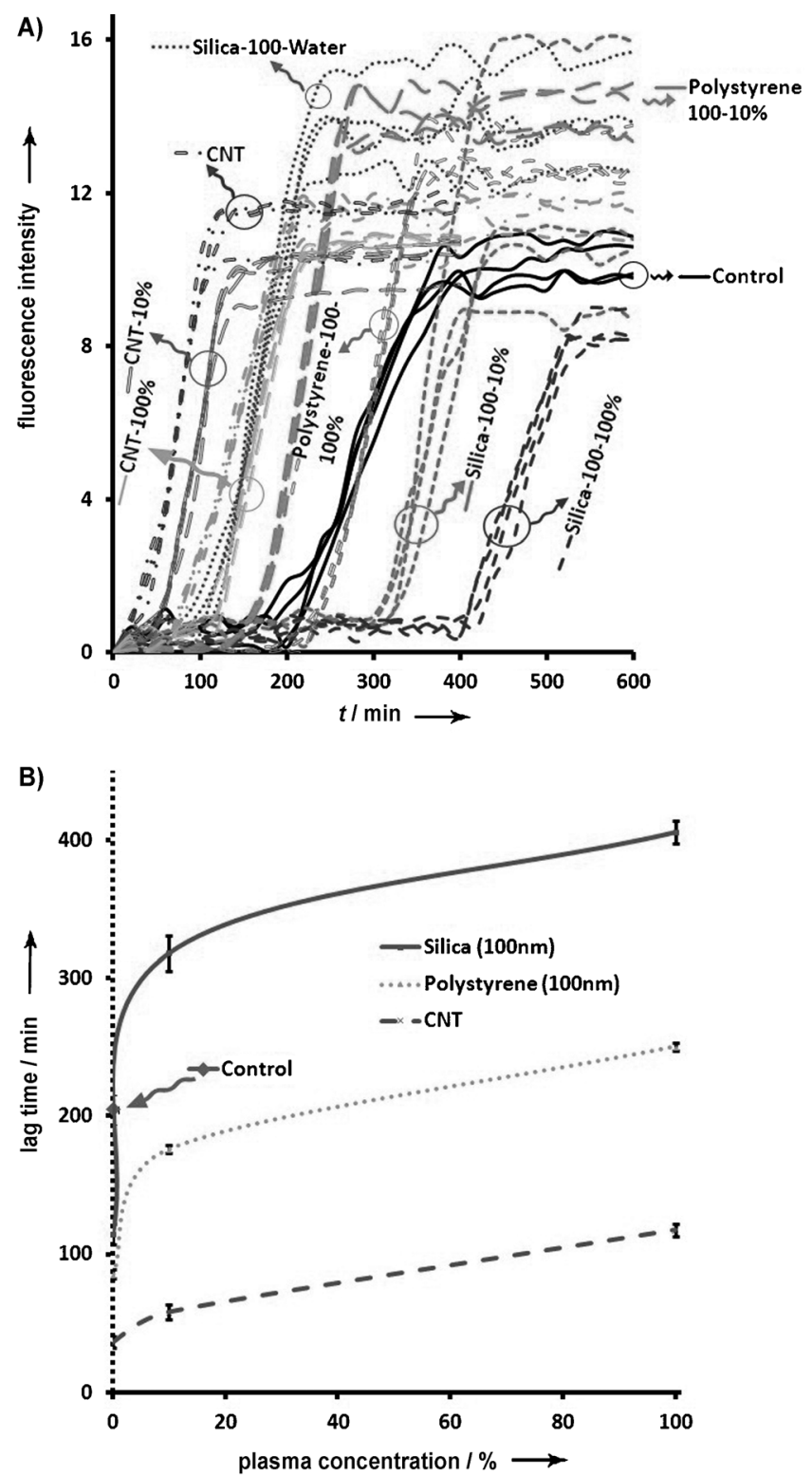

Figure 1. Kinetics of $A \beta$ fibrillation in the absence and presence of NMs with and without a protein corona. A) Fibrillation kinetics of $A \beta(5 \mu \mathrm{M})$ after incubation with pristine and protein-corona-coated NMs. B) Calculated lag times for $A \beta(5 \mu \mathrm{M})$ after incubation with pristine and protein-corona-coated NMs. a plasma protein corona it resulted in a considerable increase in the lag times for the fibrillation process, compared with that in the presence of the pristine NMs. For $100 \mathrm{~nm}$ silica NMs, fibrillation in the presence of the corona-coated NMs actually occurred more slowly than for free $A \beta$. The extent to which the lag time for $A \beta$ fibrillation increased depended on the NM-protein corona composition. Particles incubated in $100 \%$ plasma showed greater inhibitory effects than particles coated in $10 \%$ plasma, for all NMs used in this study (see Figure $1 \mathrm{~B}$ ). NMs bearing a protein corona slowed the fibrillation of $A \beta$, regardless of size, surface chemistry, charge or aspect ratio when compared with the same pristine (bare) NM. It is known that the composition and thickness of the protein corona is dependent on the ratio of the NM surface area to protein concentration, and to a lesser extent on the incubation time of the NMs and plasma proteins. ${ }^{[14]}$ In order to assess the effect of protein-NM interaction time on the $A \beta$ fibrillation process, $100 \mathrm{~nm}$ silica nanoparticles were used to probe the fibrillation process in a time-resolved manner ( 1 and $6 \mathrm{~h}$ ). The lag time for $A \beta$ fibrillation was observed to increase with both increasing plasma protein concentration and increasing incubation time. Although there was a modest increase in the lag time with NMs incubated in plasma (at both concentrations) for longer times, a much greater increase in lag time was achieved when the NM was incubated at the higher plasma concentration (Figure 2).
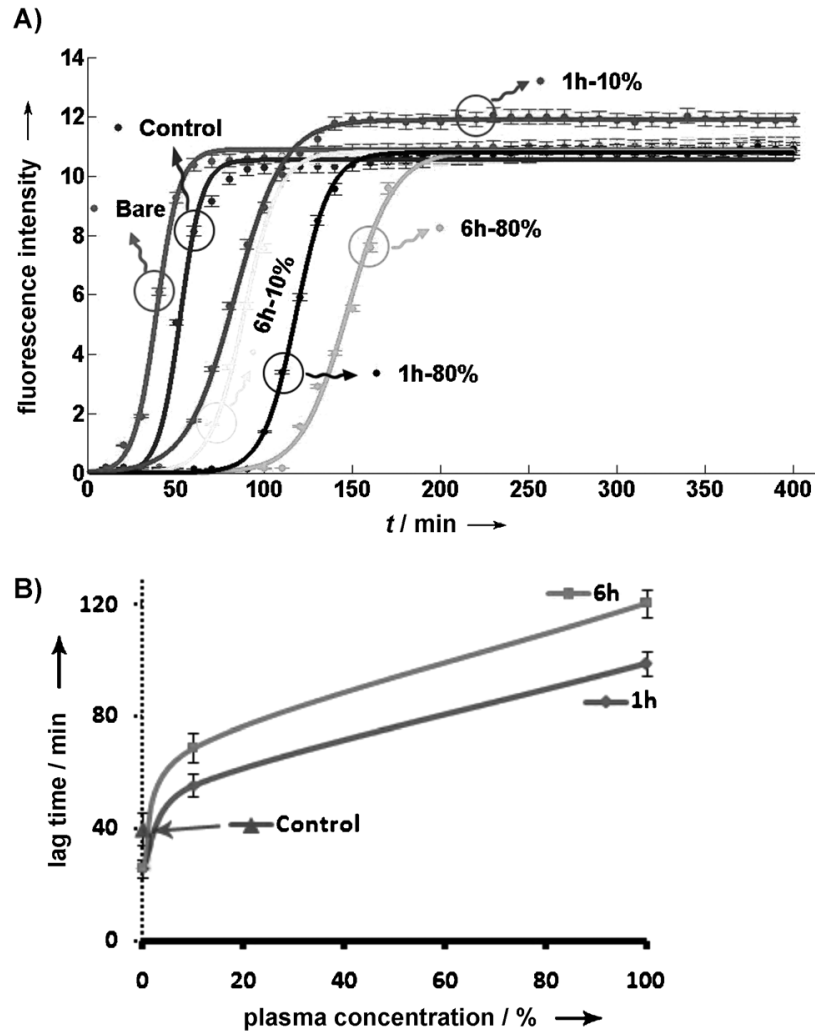

Figure 2. Effect of NM-plasma incubation time on the kinetics of $A \beta$ fibrillation ( $A \beta$ concentration: $20 \mu \mathrm{M}$ ) in the presence of NMs. A) Time-dependent ThT fluorescence was used to monitor $A \beta$ fibrillation in the absence and presence of protein-coated NMs, in a time resolved manner (e.g., $6 \mathrm{~h}-100 \%$ is the $100 \mathrm{~nm}$ silica NM preincubated with $100 \%$ plasma for a period of $6 \mathrm{~h}$ ). B) calculated lag times from (A). 
Differential centrifugal sedimentation (DCS) has been previously used to characterize nanoparticle-corona complexes. ${ }^{[14]}$ To ensure that our nanoparticles were coated with a corona consistent with what has been observed for other nanoparticle systems, DCS experiments were conducted on silica particles with and without a corona in the presence and absence of $A \beta$. The DCS results are consistent with those obtained for other NM corona systems (Figures S4 and S5). These measurements show that the main peaks of the bare $100 \mathrm{~nm}$ and $200 \mathrm{~nm}$ silica NMs were shifted slightly to lower sizes, after incubation in plasma, removal of excess plasma protein and resuspension in buffer. The decrease in observed (apparent) size is due to the change in density of the NM-corona complex relative to the bare NM, since the actual size of the particles increases, as confirmed by our previous reports. ${ }^{[14,15]}$ This apparent size decrease is consistent with results obtained previously. ${ }^{[14]}$ After addition of $A \beta$ monomers, the NM corona peak is shifted to slightly lower sizes again, consistent with the addition of $A \beta$ to the corona and a further decrease in the overall particlecorona density.

Surface curvature was not observed to play a key role in the NM-induced $A \beta$ fibrillation process: measuring the fibrillation rate of 100 and $200 \mathrm{~nm}$ silica NMs where the surface area was fixed, no change of fibrillation rate was observed. The results are shown in Figure S3. In this case, when the NM surface area exposed to $A \beta$ is fixed, there was no significant particle-size effect at either $A \beta$ concentration ( 20 or $40 \mu \mathrm{M} A \beta$ ).

In order to confirm the ThT assay data, transmission electron microscopy (TEM) of several samples was conducted. TEM images (Figures $3 A-D$ and 56 ) demonstrate that $A \beta$ oligomers
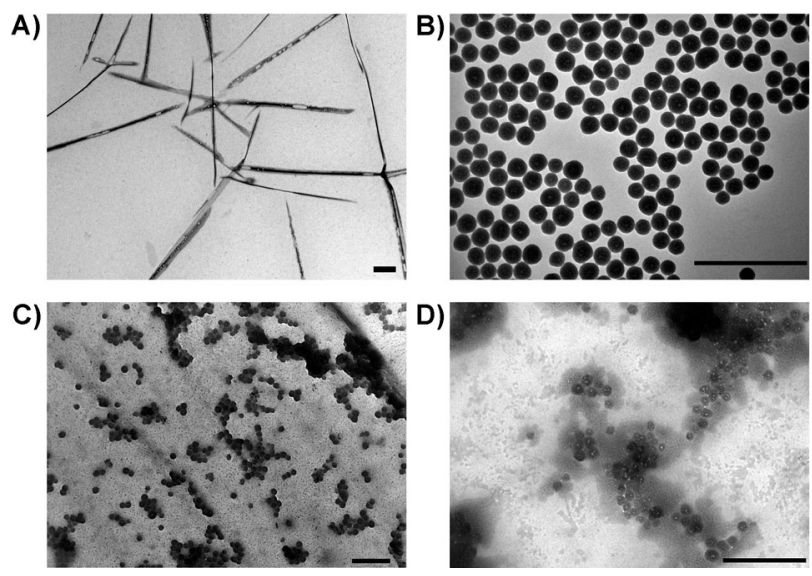

Figure 3. TEM images of A) $A \beta$ fibrils, B) bare $100 \mathrm{~nm}$ silica NMs $\left(1 \mathrm{mg} \mathrm{mL}^{-1}\right)$, C) and D) $100 \mathrm{~nm}$ corona-coated silica NMs (plasma concentrations of $10 \%$ and $100 \%$, respectively) incubated with $A \beta$. Images were taken after $6 \mathrm{~h}$ of incubation of the various NMs with $A \beta$. Scale bars: $500 \mathrm{~nm}$.

and protofibrils were formed both in the bulk solution and at the surface of protein-coated NMs. Interestingly, mature fibrils were not observed either on the corona-coated NM surface or free in solution, even after several hours of incubation (Figure $3 C$ and $D)$.
We (and others) have shown that pristine MNs can accelerate the rate of fibril formation of $A \beta$ in solution. ${ }^{[16,17]}$ We have shown here, however, that the protein corona, which is known to coat NMs in a biological environment, decreases the rate of fibril formation (measured by ThT assay and TEM). This happens for every type of NM we have tested. In the presence of silica NMs (100 and $200 \mathrm{~nm}$ ), fibrillation occurs more slowly than for $A \beta$ alone and is independent of particle size. DCS experiments indicate that $A \beta$ binds to the NM corona, and features consistent with $A \beta$ oligomers have been observed on the surface of corona-coated silica NMs by TEM (Figure S6). Notably, the TEM images also show an absence of fibrillar structures either at the corona surface or in bulk solution. Taken together, these results suggest that nucleation of $A \beta$ can and does occur on the NM surface even in the presence of a protein corona, but that growth of fibrils terminates at some point and does not proceed to the formation of mature fibrils. If indeed this is the case, the corona-coated particles, may act as a "sink" for $A \beta$, reducing the bulk concentration of the peptide and decreasing the likelihood of nucleation in the bulk while also preventing growth of fibrils on the corona surface, perhaps due to steric repulsion from the corona-coated particles, or more complex protein-protein interactions between the $A \beta$ peptide and other proteins in the corona. To test this hypothesis, coronacoated silica NMs were incubated with $A \beta$ monomer $(5 \mu \mathrm{M})$ for several hours to allow the peptide to bind to the NM corona. The particles were then recovered by gentle centrifugation and washed to remove unbound peptide. These coronacoated NMs containing preseeded $A \beta$ oligomers/protofibrils were then mixed with fresh $A \beta$ monomer $(5 \mu \mathrm{M})$, and TEM images were taken after $6 \mathrm{~h}$ (Figure 4). We see here that there is significant aggregation of the corona-coated silica NMs, probably through $A \beta$ assemblies. Interestingly, there is still an absence of mature $A \beta$ fibrils in all samples with only short fibrillar structures observed with $10 \%$ plasma-coated silica NMs and even smaller assemblies present with the $100 \%$ plasmacoated silica NMs.

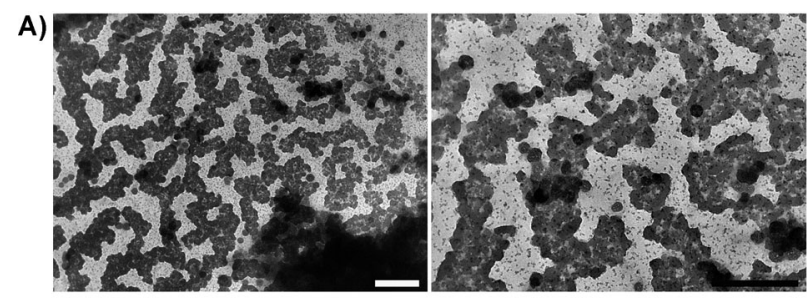

B)

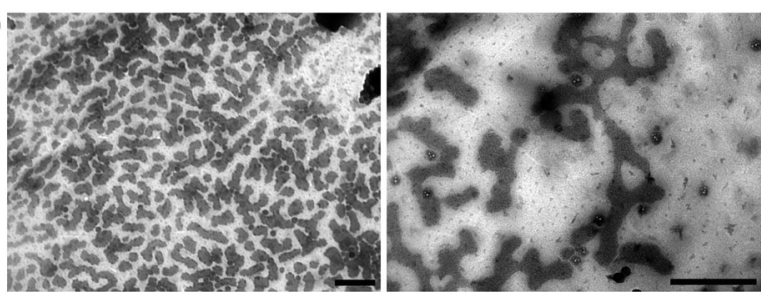

Figure 4. TEM images of $100 \mathrm{~nm}$ silica NMs incubated in A) $10 \%$ and B) $100 \%$ plasma containing $5 \mu \mathrm{M} A \beta$ where the NMs were collected by centrifugation and subsequently incubated with fresh $A \beta$ monomers $(5 \mu \mathrm{M})$ for $6 \mathrm{~h}$. Scale bars: $500 \mathrm{~nm}$. 
There is also an absence of $A \beta$ fibrils in the bulk solution, strongly suggesting that the corona-coated silica NMs are acting as a sink for $A \beta$. In order to understand why the NM corona binds to $A \beta$ and prevents growth of $A \beta$ fibrils, we need to compare its surface to that of a pristine NM surface. The pristine surface is isotropic and defined by its physico-chemical characteristics, such as size, shape, hydrophobicity and charge. The corona surface however is a highly anisotropic, heterogeneous and dynamic. It is composed of a variety of proteins, each with different affinities for $A \beta$ monomers and higher order $A \beta$ assemblies. If only some of the proteins contained in the NM corona have sufficient affinity to bind $A \beta$ monomers this will limit either the capacity of the NM to bind $A \beta$, or the ability of $A \beta$ to form higher order assemblies. Steric repulsion from proteins in the corona may also play a role in preventing addition of $A \beta$ monomers to pre-existing $A \beta$ oligomers within the NM corona. On the basis of experiments performed at $60^{\circ} \mathrm{C}$, at which the proteins were denatured (Figure S3), however, it seems that the NM protein corona must contain proteins in their native form to be effective as an inhibitor of fibril formation. Corona proteins that have been denatured by heat actually accelerate the formation of protein fibrils, mostly likely due to the exposure of hydrophobic amino acid residues from proteins that do not refold after heating. Further work will be required to understand more fully the specific mechanism by which fibril formation is inhibited by the NM protein corona.

In summary, we have shown that the presence of the NM protein corona inhibits the formation of $A \beta$ fibrils for all of the NMs investigated, whereas the same NMs accelerated the rate of $A \beta$ fibrillation when bare. DCS and TEM data suggested that there is a direct interaction of $A \beta$ with the proteins in the NM corona. $A \beta$ oligomers and protofibrils are observed both within the NM corona and in the bulk solution, but mature fibrils are absent. The protein corona creates a shell at the surface of NMs, regardless of their core composition or shape, reduces access of the $A \beta$ to the NM surface and thus impacts on the rate of $A \beta$ fibril formation. The effect is modulated by the plasma concentration used to form the NM corona, with a NM corona formed at $10 \%$ plasma (simulation of an in vitro milieu) being less effective at slowing $A \beta$ fibrillation than one formed at $100 \%$ plasma (simulation of an in vivo milieu). Several key questions emerge from these studies in the context of nanoparticle impact on protein fibrillation, which should be explored. However, we emphasize here that such investigations should be undertaken as a precautionary measure. No results reported here are sufficiently mature or sufficiently close to the in vivo conditions to suggest any particular hazard is associated with the use of NMs. Rather they constitute a desire to systematically understand the issue of nanoscale interfacial roughness and the impacts of NMs on fibrillation under increasingly realistic (biologically relevant) conditions. We note first that the NMs studied here form a relatively small set compared to those expected to appear increasingly in products in the coming years. From some of these future NMs, one many expect, significantly, various types of corona, including those that are themselves potentially profibrillar, as for example those containing unfolded proteins, and leading to an NF- $\mathrm{KB}$ response $\mathrm{e}^{[18,19]}$ and this aspect should be explored. Importantly, while for corona-nanoparticle complexes there is a reduction in the amount of protein fibrillation, there are still significant levels of oligomerisation of $A \beta$ on the NM corona surface and in the bulk solution. The implications of this in terms of potential hazard are not simple therefore. Indeed, $A \beta$ oligomers are known to be more toxic than mature fibrils, and are indeed considered by some to be important in the genesis of Alzheimer's disease. ${ }^{[20]}$

\section{Acknowledgements}

M.M. was funded through an ESF EpitopeMap RNP Exchange grant to visit CBNI, which is gratefully acknowledged. Parts of this work were conducted under the framework of the INSPIRE programme, funded by the Irish Government's Programme for Research in Third Level Institutions, Cycle 4, National Development Plan 2007-2013 (M.P.M.). Additional funding from EU FP7 Small Collaborative project NeuroNano (NMP4-SL-2008-214547, I.L.) and EU FP7 Large Collaborative project NAMDIATREAM (NMP4LA-2010-246479, F.B.) is also acknowledged. J.J.M. acknowledges financial support from the Science Foundation Ireland Stokes Lectureship. The authors acknowledge Tiina Toivonen for use of the Electron Microscopy Laboratory Centre (Conway Institue, University College Dublin). The authors also acknowledge Diego Stefani for purification of the CNT.

Keywords: fibrillation - inhibitory effect $\cdot$ nanomaterials physicochemical properties - protein corona

[1] C. M. Dobson, Nature 2003, 426, 884.

[2] a) J. D. Sipe, Annu. Rev. Biochem. 1992, 61, 947; b) L. C. Serpell, Biochim. Biophys. Acta Mol. Basis Dis. 2000, 1502, 16; c) J. Hardy, D. J. Selkoe, Science 2002, 297, 353; d) A. D. Williams, E. Portelius, I. Kheterpal, J. Guo, K. D. Cook, Y. Xu, R. Wetzel, J. Mol. Biol. 2004, 335, 833.

[3] S. B. Prusiner, Proc. Natl. Acad. Sci. USA 1998, 95, 13363.

[4] R. Khurana, C. Ionescu-Zanetti, M. Pope, J. Li, L. Nielson, M. RamirezAlvarado, L. Regan, A. L. Fink, S. A. Carter, Biophys. J. 2003, 85, 1135.

[5] S. Chen, V. Berthelier, J. B. Hamilton, B. O'Nuallain, R. Wetzel, Biochemistry 2002, 41, 7391.

[6] J. Ferkinghoff-Borg, J. Fonslet, C. B. Andersen, S. Krishna, S. Pigolotti, H. Yagi, Y. Goto, D. Otzen, M. H. Jensen, Phys. Rev. E 2010, 82, 010901.

[7] a) H. Naiki, Amyloid Int. J. Exp. Clin. Invest. 1977, 4, 223; b) V. J. McParland, A. P. Kalverda, S. W. Homans, S. E. Radford, Nat. Struc. Biol. 2002, 9, 326.

[8] L. Fei, S. Perrett, Int. J. Mol. Sci. 2009, 10, 646

[9] E. Hellstrand, B. Boland, D. M. Walsh, S. Linse, ACS Chem. Neurosci. 2010, $1,13$.

[10] a) R. Nelson, D. Eisenberg, Adv. Protein Chem. 2006, 73, 235; b) T. Sato, P. Kienlen-Campard, M. Ahmed, W. Liu, H. Li, J. I. Elliott, S. Aimoto, S. N. Constantinescu, J. N. Octave, S. O. Smith, Biochemistry 2006, 45, 5503; c) A. T. Petkova, W. M. Yau, R. Tycko, Biochemistry 2006, 45, 498.

[11] a) C. Cabaleiro-Lago, O. Szczepankiewicz, S. Linse, Langmuir 2012, 28, 1852 ; b) S. Goy-López, J. Juárez, M. Alatorre-Meda, E. Casals, V. F. Puntes, P. Taboada, V. Mosquera, Langmuir 2012, 28, 9113; c) A. Hirano, H. Yoshikawa, S. Matsushita, Y. Yamada, K. Shiraki, Langmuir 2012, 28, 3887; d) C. P. Shaw, D. A. Middleton, M. Volk, R. Lévy, ACS Nano 2012, 6, 1416.

[12] M. Mahmoudi, I. Lynch, M. R. Ejtehadi, M. P. Monopoli, F. Baldelli Bombelli, S. Laurent, Chem. Rev. 2011, 111, 5610.

[13] a) L. Xiao, D. Zhao, W.-H. Chan, M. M. F. Choi, H.-W. Li, Biomaterials 2010 31, 91; b) S. Auer, A. Trovato, M. Vendruscolo, PLoS Comput. Biol. 2009, 
5, e1000458; c) M. J. Kogan, N. G. Bastus, R. Amigo, D. Grillo-Bosch, E. Araya, A. Turiel, A. Labarta, E. Giralt, V. F. Puntes, Nano Lett. 2006, 6, 110

[14] D. Walczyk, F. Baldelli Bombelli, M. P. Monopoli, I. Lynch, K. A. Dawson, J. Am. Chem. Soc. 2010, 132, 5761.

[15] M. P. Monopoli, D. Walczyk, A. Campbell, G. Elia, I. Lynch, F. Baldelli Bombelli, K. A. Dawson, J. Am. Chem. Soc. 2011, 133, 2525.

[16] C. Cabaleiro-Lago, F. Quinlan-Pluck, I. Lynch, S. Lindman, A. M. Minogue, E. Thulin, D. M. Walsh, K. A. Dawson, S. Linse, J. Am. Chem. Soc. 2008, 130, 15437-15443.

[17] W.-h. Wu, X. Sun, Y.-p. Yu, J. Hu, L. Zhao, Q. Liu, Y.-f. Zhao, Y.-m. Li, Biochem. Biophys. Res. Commun. 2008, 373, 315-318.
[18] P. Ball, ChemPhysChem 2012, 13, 2173.

[19] Z. J. Deng, M. Liang, M. Monteiro, I. Toth, R. F. Minchin, Nat. Nanotechnol. 2011, 6, 39

[20] D. M. Walsh, I. Klyubin, J. V. Fadeeva, M. J. Rowan, D. J. Selkoe, Biochem. Soc. Trans. 2002, 30, 552.

Received: January 4, 2013

Published online on February 18, 2013 